\title{
Decitabine attenuates dextran sodium sulfate-induced ulcerative colitis through regulation of immune regulatory cells and intestinal barrier
}

\author{
CHANG SU ${ }^{1,2^{*}}$, SHAOQUN LIU ${ }^{1 *}$, XIAOYING MA ${ }^{3}$, XIAOTONG YANG ${ }^{3}$, \\ JIANWEN LIU $^{3}$, PEIYONG ZHENG ${ }^{4}$ and YIOU CAO ${ }^{1}$ \\ ${ }^{1}$ Department of Surgery, Minhang Hospital, Fudan University, Shanghai 201199; ${ }^{2}$ Department of General Surgery, \\ The Second Affiliated Hospital of Soochow University, Suzhou, Jiangsu $215004 ;{ }^{3}$ State Key Laboratory of \\ Bioreactor Engineering and Shanghai Key Laboratory of New Drug Design, School of Pharmacy, \\ East China University of Science and Technology, Shanghai 200237; ${ }^{4}$ Institute of Digestive Diseases, Longhua Hospital, \\ Shanghai University of Traditional Chinese Medicine, Shanghai 200032, P.R. China
}

Received February 2, 2020; Accepted April 9, 2020

DOI: $10.3892 /$ ijmm.2020.4605

\begin{abstract}
To investigate the effect of decitabine on the regulation of intestinal barrier function in mice with inflammatory bowel disease, an experimental model of colitis was established via drinking water with dextran sulfate sodium (DSS). Hematoxylin and eosin staining was used to observe the pathological changes of the colon. Cytokine production was measured by an ELISA assay. Flow cytometry was used to measure the level of regulatory $\mathrm{T}$ cells. Immunofluorescence, immunohistochemistry and western blot analyses detected the protein expression and distribution in colon tissue. Following the administration of decitabine, the symptoms of intestinal inflammation in the mice were significantly relieved; the expression of IL-17 was decreased, and the levels of TGF- $\beta$ and IL-10 were increased. In addition, the induction of forkhead box P3 (Foxp3) in naive T cells increased the proportion of $\mathrm{CD}^{+}{ }^{+} \mathrm{Foxp}^{+} \mathrm{T}$ cells in $\mathrm{CD}^{+} \mathrm{T}$ cells. Furthermore, decitabine increased the levels of zonular occludens-1 and occludin, and inhibited the phosphorylation of ERK1/2, JNK and p38. In conclusion, the present study suggested that decitabine could alleviate DSS-induced impaired colon barrier and the weight
\end{abstract}

Correspondence to: Dr Yiou Cao, Department of Surgery, Minhang Hospital, Fudan University, 170 Xinsong Road, Minhang, Shanghai 201199, P.R. China

E-mail: yioucao_doctor@126.com

Dr Peiyong Zheng, Institute of Digestive Diseases, Longhua Hospital, Shanghai University of Traditional Chinese Medicine, 725 Wanpingnan Road, Xuhui, Shanghai 200032, P.R. China

E-mail: zpychina@sina.com

${ }^{*}$ Contributed equally

Key words: decitabine, dextran sulfate sodium, mitogen-activated protein kinase, inflammatory bowel disease loss, mucus and bloody stools in mice by releasing the inhibitory factor IL-10, reducing the pro-inflammatory factor IL-17, activating $\mathrm{CD}^{+}{ }^{+}$Foxp $3^{+} \mathrm{T}$ cells and inhibiting the activation of the MAPK pathway.

\section{Introduction}

Inflammatory bowel disease (IBD) is a chronic non-specific inflammatory disease that occurs in the gastrointestinal tract, which includes ulcerative colitis (UC) and Crohn's disease (1). The pathogenesis of IBD has not yet been fully elucidated, however, it is currently understood that intestinal mucosal immune overreaction and dysfunction are the main causes (2). Current strategies for treating IBD include anti-inflammatory drugs and immunomodulators (3). Hartnett and Egan (4) has shown that patients with IBD have a lifetime risk of colorectal cancer that is two to three times higher than the general population. Molodecky et al (5) reported an increasing incidence and prevalence of the inflammatory bowel diseases with age. Ramos and Papadakis (6) found that inflammatory bowel diseases were associated with microbial dysbiosis. The number of methylated genes in non-neoplastic colonic mucosa could predict colorectal cancer (CRC) with good accuracy for both non-inflammatory and inflammatory-related CRC (7). DNA methylation is an epigenetic change that occurs on the cytosine of genomic $\mathrm{CpG}$ dinucleotides and plays an important role in the regulation of IBD gene expression (8). Covalent methylation of DNA CpG islands is catalyzed by methyltransferases, which methylate C-5 of cytosine nucleotides (9). The global cytosine methylation pattern in the mammalian genome appears to be established by the complex interaction of at least three independently encoded DNA methyltransferases (DNMT): DNMT1, DNMT3A and DNMT3B (1). The expression levels of DNMT1 and DNMT3A are significantly increased in UC-related carcinogenesis compared with non-inflammatory colorectal carcinogenesis (10).

The DNMT inhibitor decitabine (5-aza-2'-deoxycytidine) is a 5-azacytidine deoxyribose analog and is currently used to 
treat hematological malignancies, including myelodysplastic syndrome, acute myeloid leukemia and chronic myelomonocytic leukemia (10). Decitabine promotes the reduction of DNA methylation and induces gene expression and differentiation. Decitabine exhibits immunomodulatory potential both in vitro and in vivo and induces demethylation of the forkhead box P3 (Foxp3) gene (11).

To mimic human IBD, the present study established an experimental colitis model by administering drinking water with $3 \%$ dextran sulfate sodium (DSS) to BALB/c mice for 7 consecutive days. The effect of the methyltransferase inhibitor decitabine on the intestinal barrier function of mice with IBD and its potential mechanism was investigated, and a theoretical basis for clinical induction of immune tolerance in the treatment of IBD was provided.

\section{Materials and methods}

Animal modeling. A total of 24 six-week-old male BALB/c mice were purchased from Shanghai Jiesijie Experimental Animal Co., Ltd. Mice were raised with a constant temperature of $23 \pm 2^{\circ} \mathrm{C}, 50 \%$ humidity, a $12 / 12 \mathrm{~h}$ light/dark cycle and ad libitum access to food and water. The animal protocol in this work was in accordance with guidelines for the care and use of laboratory animals authorized by the Medical Ethics Committee of Minhang Hospital, Shanghai, China. The animal research was approved by the Ethics Committee of Minhang Hospital, Shanghai, China [Medical Ethics Committee (2018) Approval no. 2]. A total of 24 mice were randomly divided into four groups ( $n=6$ per group). Three groups of mice were used as experimental colitis models and one group of mice served as normal controls. An experimental colitis model was established by supplementing BALB/c mice with 3\% (w/v) DSS (Sigma-Aldrich; Merck KGaA) in drinking water once a day for 7 days. On the 8th day, the decitabine, sulfasalazine (SASP) positive control and model groups were intraperitoneally administered with decitabine (Merck $\mathrm{KGaA}$; cat. no. 2353-33-5; 0.5 mg/kg), SASP (Merck KGaA; cat. no. 599-79-1; $100 \mathrm{mg} / \mathrm{kg})$ and $1 \%$ DMSO $(1 \mu \mathrm{l} / 20 \mathrm{~g})$, respectively, for 7 consecutive days. Mice were injected twice a day at an interval of $12 \mathrm{~h}$. During the experiment, no mice died unexpectedly. From the day of treating with DSS changes in body weight, external characteristics and clinical symptoms of the mice were monitored every day. The combination of these indicators can reflect the pain and suffering experienced by the animals during the experiment (12). Mice were euthanized with an intraperitoneal injection of $180 \mathrm{mg} / \mathrm{kg}$ sodium pentobarbital, and then the heartbeat of the mice was examined. The duration of animal experiments was 2 weeks from the first day of treatment with DSS to euthanasia.

Mouse disease activity index score. Mice of each group were examined daily for body mass, diarrhea and blood in the stool. Disease activity index (DAI) was calculated as follows: DAI $=($ weight loss score + fecal trait score + fecal occult blood score)/3) (13).

Gross morphological examination of colon tissue. After the animals were sacrificed on the 8th day, the entire colonic intestine from the anus to the end of the cecum was isolated.
Following washing, the extent of inflammation and ulceration was assessed and the length of the colon was recorded. Colon gross morphological damage index (CMDI) score was calculated as reported previously (1).

Hematoxylin and eosin $(H \& E)$ staining. Distal colon tissue $(1 \mathrm{~cm})$ was fixed in $4 \%$ paraformaldehyde for $24 \mathrm{~h}$ at room temperature, embedded in paraffin, sectioned to a thickness of $8-\mu \mathrm{m}$, and stained with H\&E for $1 \mathrm{~min}$ at room temperature. Histopathological changes were observed under a light microscope (magnification, $\mathrm{x} 20$ or $\mathrm{x} 40$ ) and scored according to the criteria described by Andújar et al (14). The colon histopathology index (CHPI) was determined as previously described (15).

ELISA. Since the effect of DSS is more pronounced at the distal end compared with the proximal colon (16), small sections $(\sim 1 \mathrm{~cm})$ of excised distal colonic tissue were collected for ELISA and western blot assays. Subsequently, $\sim 10 \mathrm{mg}$ colon tissue of each group was collected and the tissue was homogenized with $1 \mathrm{ml}$ PBS (pH, 6.0; containing $1 \mu \mathrm{g}$ aprotinin (Shanghai Qcbio Science \& Technologies Co. Ltd.; cat. no. 20105ES08) and $1 \mu \mathrm{g}$ leupeptin pepstatin A (Maokangbio Co. Ltd.; cat. no. 103476-89-7). After homogenate liquid was centrifuged at $16,099 \times \mathrm{g}$ for $20 \mathrm{~min}$ at $4^{\circ} \mathrm{C}, 500 \mu \mathrm{l}$ supernatant was collected for ELISA. ELISA kits for IL-17 (Boster Biological Technology; cat. no. EK0431), TGF- $\beta$ (Beijing Solarbio Science \& Technology Co., Ltd.; cat. no. SEKM-0035) and IL-10 (Boster Biological Technology; cat. no. EK0417) were used to measure the cytokine levels, according to the manufacturer's instructions.

Flow cytometry analysis. The spleens of the mice were cut, filtered through a $200-\mu \mathrm{m}$ nylon mesh, centrifuged at $251.55 \mathrm{x} \mathrm{g}$ for $5 \mathrm{~min}$ at $4^{\circ} \mathrm{C}$, and then the supernatant was removed. $\mathrm{NH}_{4} \mathrm{Cl}(2 \mathrm{ml} ; 0.83 \%)$ was added to lyse the red blood cells. After $2 \mathrm{~min}, 15 \mathrm{ml}$ PBS was added to terminate the lysis. The cell density was adjusted to $1 \times 10^{6}$ cells $/ \mathrm{ml}$ in PBS and the cells were blocked with $2.5 \% \mathrm{BSA}$ at room temperature for $1 \mathrm{~h}$. Subsequently, the cells were incubated with FITC-rat anti-mouse CD4 (cat. no RM4-5; eBioscience; Thermo Fisher Scientific, Inc.) in $2.5 \% \mathrm{BSA}$ for $1 \mathrm{~h}$ at room temperature in the dark and washed twice with PBS. Cells were then fixed with fixation reagent (cat. no. 00-5523-00; eBioscience; Thermo Fisher Scientific, Inc.) for $15 \mathrm{~min}$ at room temperature and permeabilized for intracellular staining. Subsequently, $1 \mathrm{ml}$ of a 3:1 permeabilization reagent (cat. no. 00-5523-00; eBioscience; Thermo Fisher Scientific, Inc.) was added, and the cells were incubated at $4^{\circ} \mathrm{C}$ for $1 \mathrm{~h}$ in the dark, followed by the addition of cell permeabilization buffer ( $2 \mathrm{ml}$; cat. no. $39487 \mathrm{~s}$; Cell Signaling Technology, Inc.), centrifugation twice, and the resuspension of cells to a volume of $100 \mu \mathrm{l}$. PE-rat anti-mouse Foxp3 (cat. no. 72-5775-40; eBioscience; Thermo Fisher Scientific, Inc.) in $2.5 \%$ BSA was added and incubated for $1 \mathrm{~h}$ at $4^{\circ} \mathrm{C}$ in the dark. Cell phenotypes were detected using a flow cytometer and the data were analyzed using FlowJo software (FlowJo LLC; version 7.6.1).

Immunofluorescence. Sections were blocked in 5\% BSA for $1 \mathrm{~h}$ at room temperature and were stained with anti-mouse 
antibodies against zonular occludens-1 (1:1,000; ZO-1; Novus Biologicals, Ltd.; cat. no. NBP1-85047) and occludin (1:200; Abcam; cat. no. ab216327) in $2.5 \%$ BSA for overnight at $4^{\circ} \mathrm{C}$, and then with the secondary antibody goat anti-rabbit IgG $(\mathrm{H}+\mathrm{L})$ with a FITC fluorescent label $(1: 1,000$; GeneCopoeia; cat. no. L147A) or Cy-3 tag (1:1,000; Boster Biological Technology; cat. no. BA1032) in 2.5\% BSA for $2 \mathrm{~h}$ at room temperature. Sections were examined under a fluorescence microscope (magnification, x20). Quantitative analysis was conducted using Image $\mathbf{J}$ software (National Institutes of Health; version 1.46).

Immunohistochemistry. ZO-1 and occludin anti-mouse antibodies were diluted with PBS at a ratio of 1:100; and PBS was used as a negative control for overnight at $4^{\circ} \mathrm{C}$. Subsequently, eight fields of view were randomly observed under a fluorescence microscope (magnification, $\mathrm{x} 40$ ), and the percentage of positive cells and the intensity of staining was scored. The percentage of stained cells in the field of view was scored as follows: $<5 \%, 0$ point; $5-25 \%, 1$ point; $26-50 \%, 2$ points; $51-75 \%, 3$ points; and $>75 \%, 4$ points. The staining intensity was scored as follows: No staining, 0 point; light yellow staining, 1 point; brown staining, 2 points; and tan staining, 3 points. The two scores were then multiplied to calculate the immunoreactivity score.

Western blotting. For western blotting, 100 mg distal colonic tissue was weighed and cut into pieces. The tissue was then grinded with liquid nitrogen and added to $1 \mathrm{ml}$ ice-cold PBS in a round bottom flask. The tissue was homogenized at a speed of $90.56 \mathrm{x} \mathrm{g}$ and transferred to a $1.5 \mathrm{ml} \mathrm{EP}$ tube. The homogenate liquid was washed twice with PBS containing PMSF (Beijing Solarbio Science \& Technology, Co., Ltd.) and then centrifuged at $16,099 \mathrm{x}$ g for $5 \mathrm{~min}$ at $4^{\circ} \mathrm{C}$. The supernatant was removed and $500 \mu 1$ protein lysis buffer (Beyotime Institute of Biotechnology; cat. no. P0013) was added. After the tissue was lysed on ice for $2 \mathrm{~h}$, it was centrifuged at 16,099 $\mathrm{x}$ g for $30 \mathrm{~min}$ at $4^{\circ} \mathrm{C}$. The supernatant was collected and mixed with $5 \mathrm{X}$ loading buffer (Beyotime Institute of Biotechnology) and then the protein was denatured at $100^{\circ} \mathrm{C}$ for $10 \mathrm{~min}$. Protein concentration was detected using a BCA kit (Beyotime Institute of Biotechnology) and $30 \mu \mathrm{g}$ protein was separated on a $10 \%$ SDS-PAGE gel before being transferred to PVDF membranes (Thermo Fisher Scientific, Inc.). Membranes were blocked with $4 \%$ BSA-TBST for $90 \mathrm{~min}$ and probed overnight at $4^{\circ} \mathrm{C}$ with primary antibodies in 5\% BSA against the following ZO-1 (1:2,500; Novus Biologicals, Ltd.; cat. no. NBP1-85047), occludin (1:1,000; Abcam; cat. no ab216327), ERK1/2 (1:1,000; Abways Technology; cat. no. CY1066), phosphorylated (p)-Erk1/2 (1:1,000; Abways Technology; cat. no. CY6190), JNK (1:1,000; Abways Technology; cat. no. AB3296), p-JNK (1:1,000; Abways Technology; cat. no. CY6315), p38 (1:1,000; Abways Technology; cat. no. AB3374) and p-p38 (1:1,000; Abways Technology; cat. no. CY6390). GAPDH (1:1,000; Santa Cruz Biotechnology, Inc.; cat. no. sc-166574) was used as a loading control. Then, the membranes were incubated with an anti-rabbit HRP secondary antibody (1:1,000; Jackson ImmunoResearch Laboratories, Inc.; cat. no. 111-005-003) for $2 \mathrm{~h}$ at room temperature and bands were visualized using super ECL detection reagent (Shanghai Yeasen Biotechnology, Co.,
Ltd.; cat. no. 36208ES60). Quantitative analysis was conducted using Image $\mathbf{J}$ software (National Institutes of Health; version 1.46), and the ratio of phosphorylated protein/total protein was evaluated.

Statistical analysis. Statistical analysis was performed using GraphPad Prism 5.0 software (GraphPad Software, Inc.). The data are presented as the mean \pm standard deviation. Significant differences for between multiple groups were analyzed by one-way ANOVA followed by Tukey's post hoc test. $\mathrm{P}<0.05$ was considered to indicate a statistically significant difference. Each experiment was repeated a minimum of three times.

\section{Results}

Effects of decitabine treatment on intestinal pathology and histopathology in mice. Decitabine, also known as 5-aza-2'-deoxycytidine, is a natural adenosine analog of 2'-deoxycytidine (17), and its structure is presented in Fig. 1A. Apart from the normal control group, all other BALB/c mice were given $3 \%$ DSS for 7 consecutive days to establish an experimental colitis model. On the 8th day, decitabine $(0.5 \mathrm{mg} / \mathrm{kg})$, SASP $(100 \mathrm{mg} / \mathrm{kg})$ or placebo (1\% DMSO) were intraperitoneally injected for a further 7 consecutive days. The recommended clinical dose of decitabine is $15 \mathrm{mg} / \mathrm{m}^{2}$ (18). The Meeh-Rubner formula (19) calculates the body surface area of mice. The body surface area of $20 \mathrm{~g}$ mice is $\sim 0.67 \mathrm{dm}^{2}$ (20). Thus, the mice were intraperitoneally injected with decitabine at a dose of $5 \mathrm{mg} / \mathrm{kg}$, which was only used in the preliminary experiments. A total of one week after the first treatment with decitabine, two mice in the decitabine group died; therefore, the administration concentration was reduced by ten times to $0.5 \mathrm{mg} / \mathrm{kg}$ (Fig. 1B).

The DAI was used to evaluate the therapeutic effect of decitabine on DSS-induced IBD. No mice died during the modeling process. After $24 \mathrm{~h}$ of modeling, which was after the animals were treated with DSS, the animals developed symptoms such as loose stools, severe perianal contamination, reduced food intake and weight loss. During the whole administration period, the DAI of the normal control group was 0 . Following the last day of DSS administration, the mice in the model group slightly recovered over the following three days but then their condition began to decline on the fourth day; they ate and drank less, the body weight declined and they had loose stools with mucus and pus. In the SASP group, the general condition of the mice gradually improved, and the DAI score gradually decreased. After 7 days of treatment with SASP, the symptoms of IBD were relieved and the mean DAI score was only 0.73 points. On day 4 of treatment with decitabine, food intake increased and the extent of loose stools was reduced. Compared with the model group, the body weight was increased in the decitabine group. After 7 days of treatment with decitabine, the mean average DAI score reached 0.8. (Fig. 1C).

From the results of colon length measurement, the colon length of the model group was shorter than that of the normal control group. The shortening of the colon length in mice was relieved in the SASP and decitabine groups. The general morphology of the colon and the CMDI score demonstrated that the normal control group had a smooth colon surface 



Figure 1. Effects of decitabine treatment on intestinal pathology and histopathology in mice. (A) The structure of the decitabine. (B) Modeling and administration scheme. Following successful modeling, (C) DAI and (D) CMDI scores were calculated. (E) H\&E staining. The pathological sections were imaged using a 20X objective. Eight fields were randomly selected for scoring and the combined histology score was calculated according to the formula. Data are presented as the mean \pm standard deviation. ${ }^{*} \mathrm{P}<0.05,{ }^{* *} \mathrm{P}<0.01,{ }^{* * *} \mathrm{P}<0.001$. DSS, dextran sulfate sodium; SASP, sulfasalazine; DAI, disease activity index; CMDI, colon gross morphological damage index.

and a clear texture. In the DSS alone group, the surface of the intestine was uneven, and the phenomenon of inflammatory congestion and edema. In the model group, severe inflammatory hyperemia and edema were observed in the colon and the CMDI score was significantly higher compared the normal control group $(\mathrm{P}<0.01)$. Local hyperemia and edema were observed in the decitabine intervention group, but mainly concentrated in the distal colon, and the CMDI score was significantly lower compared with in the model group $(\mathrm{P}<0.05$; Fig. 1D).

Histopathological observation and the CHPI score demonstrated that there was no obvious inflammatory cell infiltration in the normal control group, and the intact mucosal epithelium was well arranged. In the model group, the colonic mucosa was exfoliated, the gland structure was severely damaged, the gland was disordered and a large number of lymphocytes and neutrophils were infiltrated, and the CHPI score was significantly higher compared with the normal group $(\mathrm{P}<0.01)$. The infiltration of inflammatory cells, hyperplasia and edema in was alleviated in the SASP group, and the CHPI score was significantly reduced compared with the model group $(\mathrm{P}<0.001)$. In the decitabine group, the intestinal mucosa of the mice was intact, inflammatory cell infiltration was low and the CHPI score was significantly decreased compared with the model group $(\mathrm{P}<0.001$; Fig. 1E).

Expression of regulatory $T$ cell (Treg)-associated cytokines $I L-17, T G F-\beta$ and IL-10 in mice colon tissue. Tregs are important for the homeostasis of the immune system (21). CD4 ${ }^{+}$ CD25 $5^{+}$Tregs are a subset of Tregs that maintain immune 

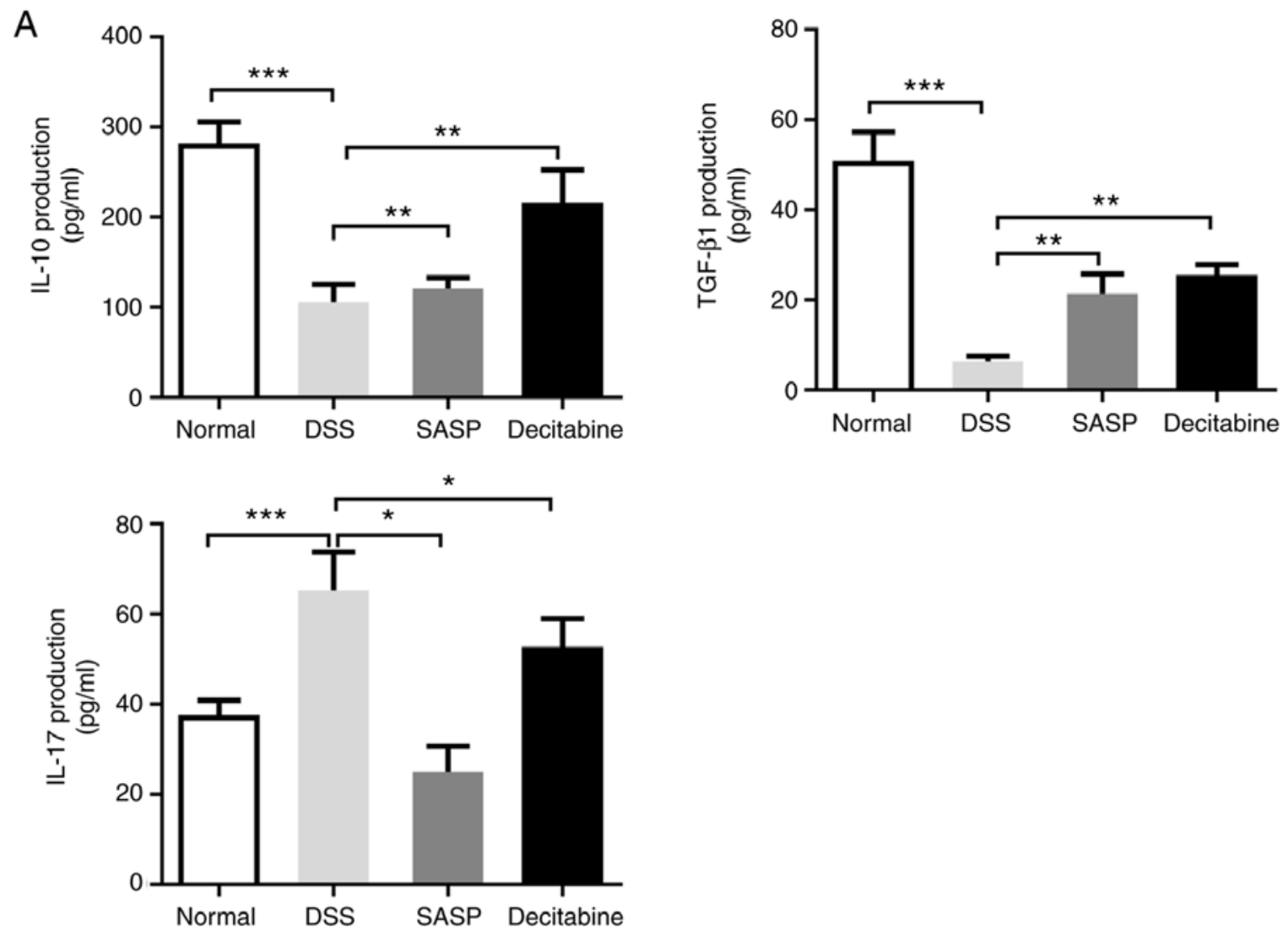

B

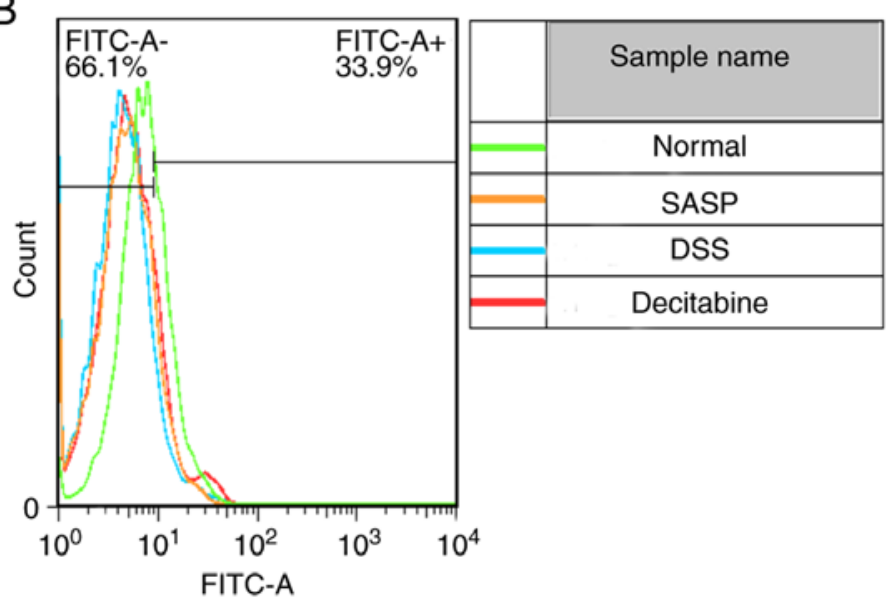



Normal SASP Decitabine

Figure 2. Th17/Treg-associated cytokine IL-17 expression is decreased and TGF- $\beta$ and IL-10 are increased following decitabine treatment. (A) ELISA detected the secretion of IL-17, TGF- $\beta$ and IL-10 cytokines in colon tissue. (B) Flow cytometry analysis of the effect of decitabine on Foxp3 in the spleen. Data are presented as the mean \pm standard deviation. ${ }^{*} \mathrm{P}<0.05,{ }^{* *} \mathrm{P}<0.01,{ }^{* * *} \mathrm{P}<0.001$. DSS, dextran sulfate sodium; SASP, sulfasalazine; FoxP3, forkhead box $\mathrm{P}$.

tolerance through direct contact with effector $\mathrm{T}$ cells and secretion of TGF- $\beta$, IL-10 and other cytokines (22). Compared with the normal control group, the level of cytokine IL-17 in the colon tissue of the model group was significantly increased, while the levels of cytokines IL-10 and TGF- $\beta$ were significantly decreased $(\mathrm{P}<0.001)$. Compared with the model group, the level of IL-17 in the SASP group was significantly lower $(\mathrm{P}<0.05)$ and the IL-10 level was significantly increased $(\mathrm{P}<0.01)$. Compared with the model group, the TGF- $\beta$ levels were significantly increased $(\mathrm{P}<0.001)$. There was a significant increase of IL-10 in the decitabine group compared with the model group $(\mathrm{P}<0.01$; Fig. $2 \mathrm{~A})$.
In the present study, Foxp3 expression in the spleen of the model group was significantly decreased compared with the normal control group $(\mathrm{P}<0.01)$, while treatments with SASP or decitabine resulted in significant increases in Foxp3 expression $(\mathrm{P}<0.01$ and $\mathrm{P}<0.001$, respectively; Fig. $2 \mathrm{~B})$.

Decitabine treatment increases the proportion of $\mathrm{CD}^{+}$ Foxp $^{+} \mathrm{T}$ cells in $\mathrm{CD}^{+} \mathrm{T}$ cells. DSS can promote edema and inflammation, and significantly increases the weight of mice spleens $(\mathrm{P}<0.001)(23)$. SASP reduced the weight of the mice spleens compared with the model group $(\mathrm{P}<0.001)$. In addition. decitabine reduced the weight of the mice spleen, but 

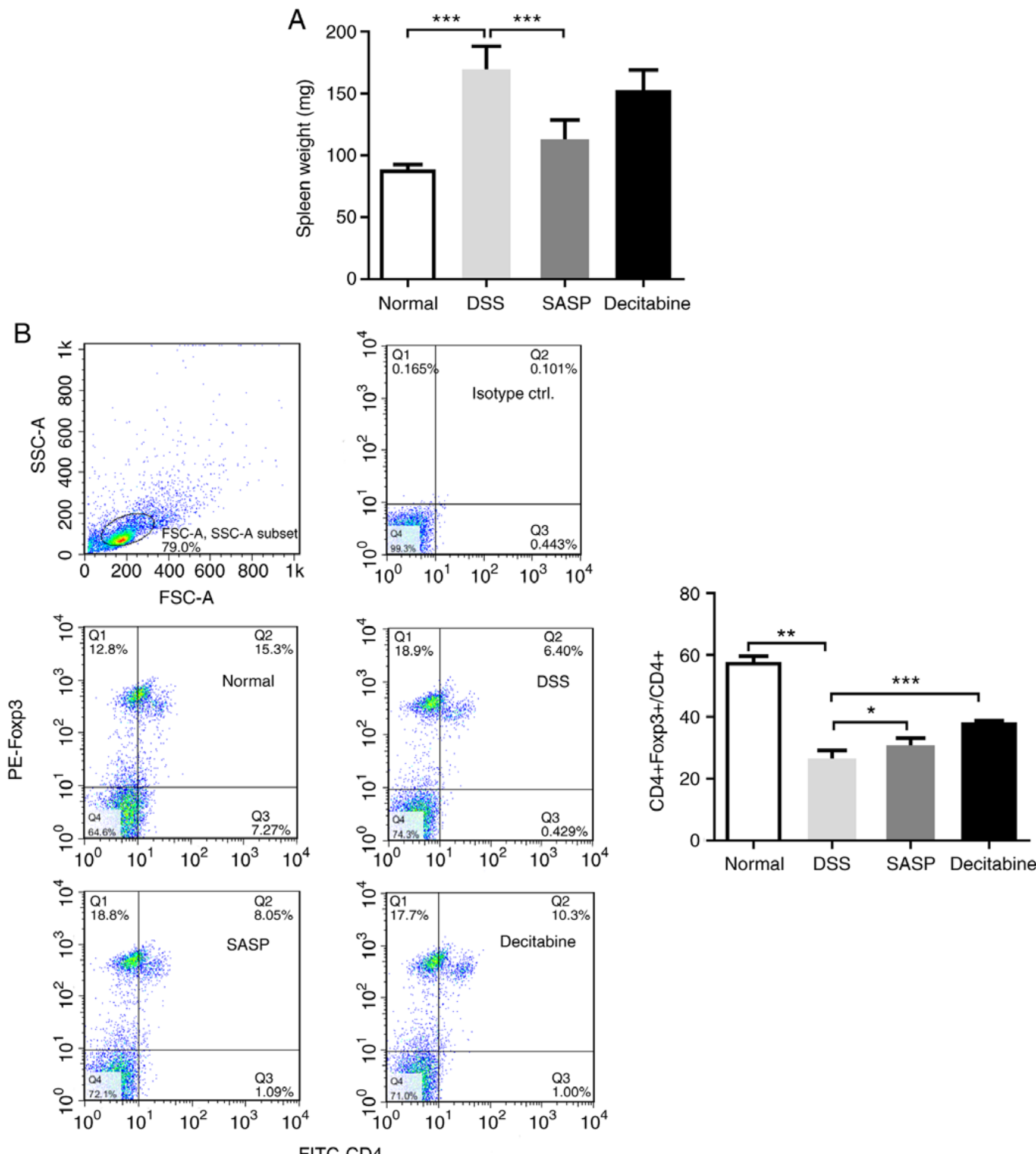

\section{FITC-CD4}

Figure 3. Decitabine treatment increases the proportion of CD4+ Foxp3+ T cells among CD4+ T cells in the spleen. (A) Mice spleen weights following treatments. (B) Flow cytometry analysis of the proportion of CD4+ Foxp3+ T cells in CD4+ T cells. Data are presented as the mean \pm standard deviation. " $\mathrm{P}<0.05$, ${ }^{* *} \mathrm{P}<0.01,{ }^{* * *} \mathrm{P}<0.001$. DSS, dextran sulfate sodium; SASP, sulfasalazine; FoxP3, forkhead box P3.

it was not statistically significant compared with the model group (Fig. 3A).

Compared with the normal control group, DSS significantly reduced the proportion of $\mathrm{CD}^{+}{ }^{+} \mathrm{Foxp}^{+} \mathrm{T}$ cells in $\mathrm{CD} 4^{+} \mathrm{T}$ cells in the spleen of mice $(\mathrm{P}<0.01)$. Compared with the model group, the proportion of $\mathrm{CD} 4^{+} \mathrm{Foxp}^{+} \mathrm{T}$ cells in $\mathrm{CD} 4^{+} \mathrm{T}$ cells was significantly increased following 7 days of intraperitoneal injection of decitabine $(\mathrm{P}<0.001$; Fig. 3B). This indicates that intraperitoneal injection of decitabine can promote the re-expression of Foxp3 in naive T cells of mice spleen, and increase the proportion of $\mathrm{CD}^{+} \mathrm{Foxp}^{+} \mathrm{T}$ cells in $\mathrm{CD} 4^{+} \mathrm{T}$ cells, suggesting that the use of methyltransferase inhibitors in vivo can play a role in demethylation and amplify Tregs.

Expression and distribution of $\mathrm{ZO}-1$ and occludin proteins in the colon tissue of mice. After the mice were sacrificed, 
A

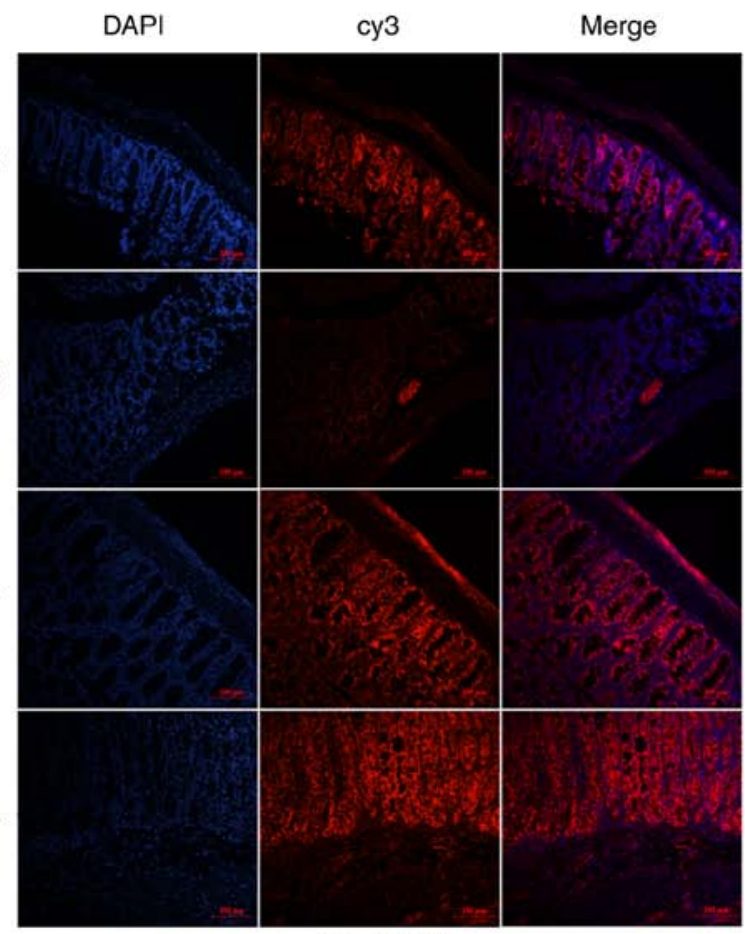

$\mathrm{ZO}-1(\mathrm{x} 20)$

B

Normal

DAPI

FITC
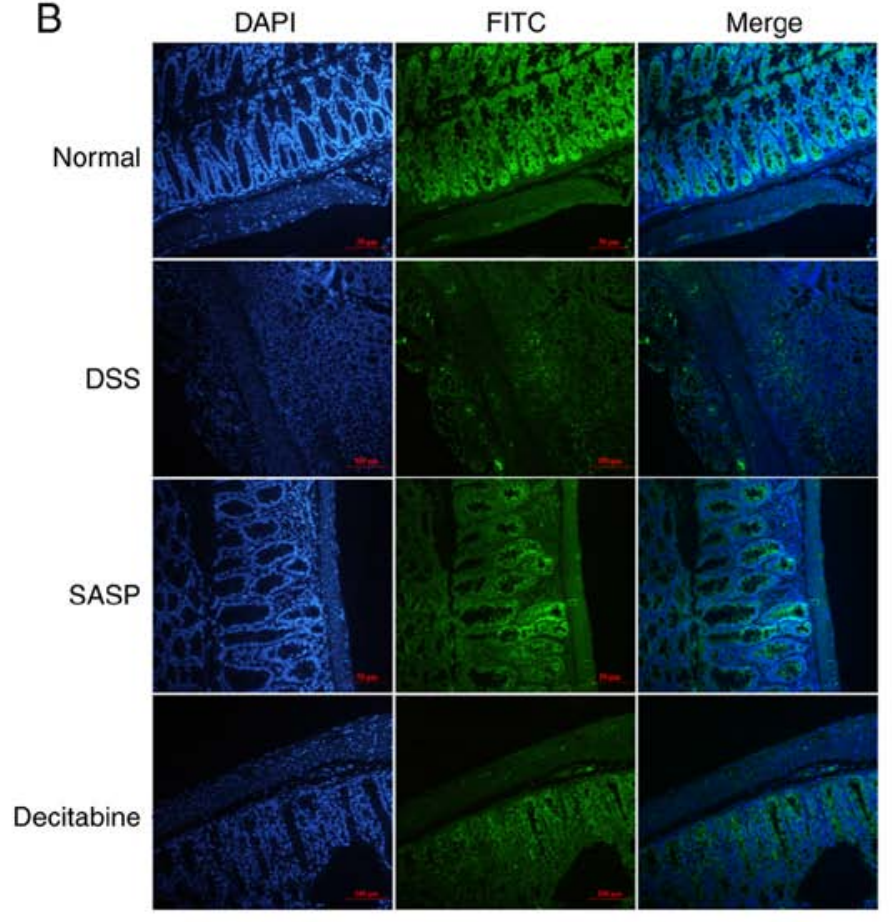

Occludin $(\times 20)$
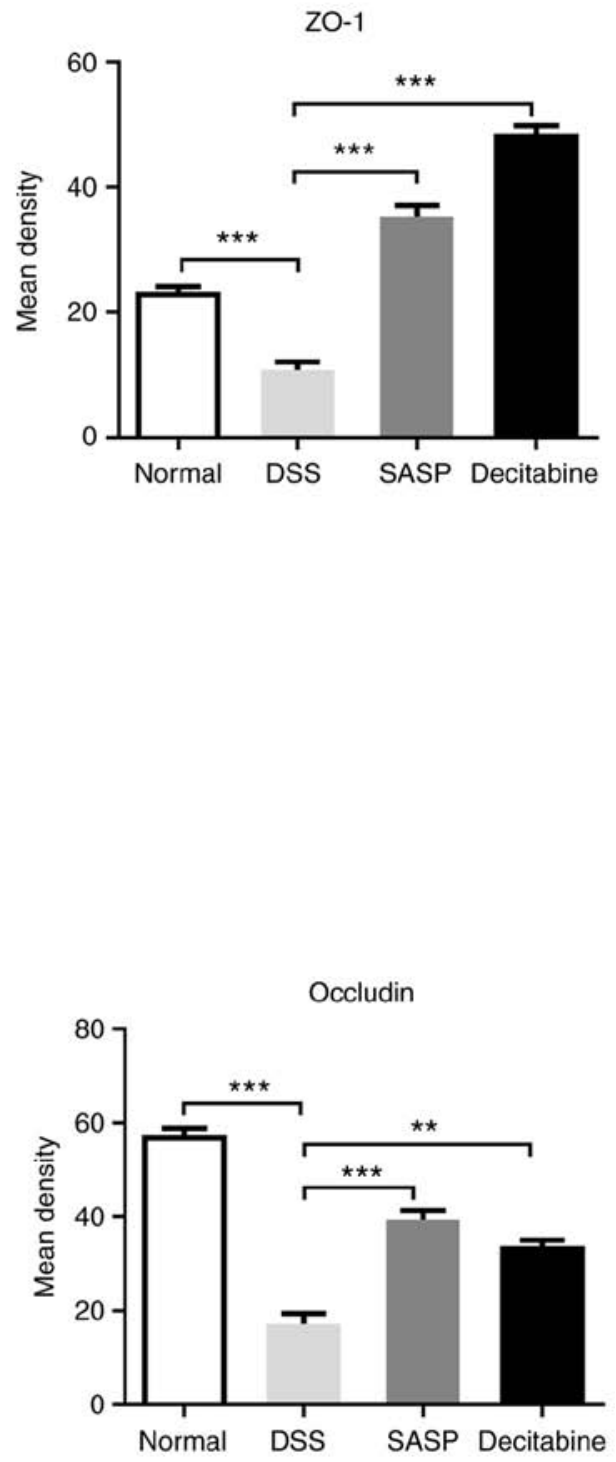

Figure 4. Expression and distribution of ZO-1 and occludin proteins in mice colon tissues according to fluorescence microscopy. Fluorescence microscopy detected the distribution of (A) ZO-1-cy3 and (B) occludin-FITC in colon tissue. Magnification, x20. Eight fields were randomly selected for quantitative analysis. Data are presented as the mean \pm standard deviation. ${ }^{* * *} \mathrm{P}<0.01,{ }^{* * * *} \mathrm{P}<0.001$. DSS, dextran sulfate sodium; SASP, sulfasalazine; ZO-1, zonular occludens-1.

the fluorescence staining of ZO-1 and occludin in the colonic mucosa of the normal control group was continuously distributed at the junction of the colon cells, and the edges were smooth and the fluorescence scope was wide. The mice in the model group demonstrated both a decreased fluorescence intensity and discontinuity distribution of tight junction proteins in the cell apical membrane. Compared with the model group, the tight junction proteins in SASP group and decitabine group were continuously distributed in the apical membrane of intestinal epithelial cells, the edges were smooth, and the fluorescence area and intensity were increased (Fig. 4A and B). The immunohistochemistry demonstrated that the intestinal epithelial structure of the normal control group was intact, while in the model group the tight junction structure and microvilli were destroyed; the gap between cells was widened and the cells were vacuolated. 
A


$\mathrm{ZO}-1$

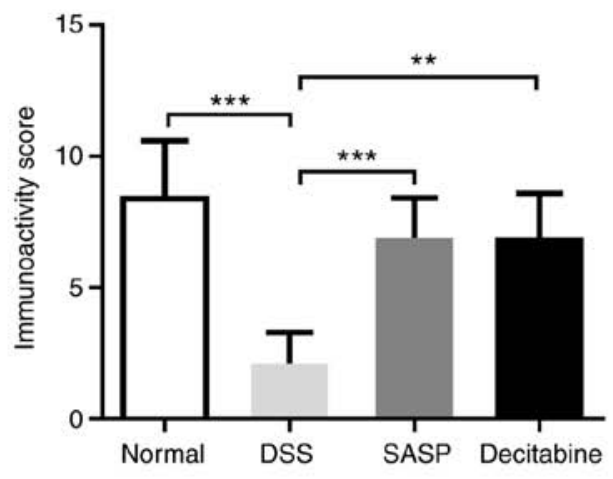

B
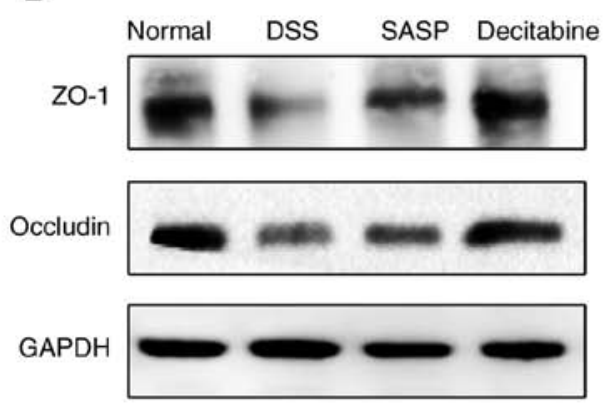

SASP
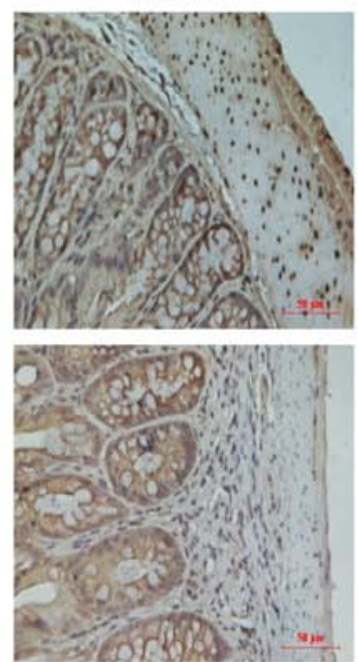

Occludin


Figure 5. Expression and distribution of ZO-1 and Occludin proteins in mice colon tissue according to immunohistochemistry. (A) Immunohistochemistry detected the distribution of ZO-1 and occludin in colon tissue. Magnification, $\mathrm{x} 40$. Eight fields were randomly collected under the microscope, and the percentage of positive cells and the intensity of staining were scored. ${ }^{* *} \mathrm{P}<0.01,{ }^{* * *} \mathrm{P}<0.001$. (B) Western blot assay detected the levels of ZO-1 and occludin in colon tissue. The results were normalized to GAPDH, and experiments were conducted at least three times. Data are presented as the mean \pm standard deviation. ${ }^{* * *} \mathrm{P}<0.001$ vs. normal. ${ }^{\# \#} \mathrm{P}<0.001$ vs. DSS. DSS, dextran sulfate sodium; SASP, sulfasalazine; ZO-1, zonular occludens-1.

As observed by immunofluorescence, the expression of ZO-1 was significantly lower in the model group compared with the normal control group, and significantly higher in the SASP $(\mathrm{P}<0.001)$ and decitabine groups $(\mathrm{P}<0.001)$ compared with the model group (Fig. 4A). As observed by immunofluorescence, the expression of Occludin was significantly lower in the model group compared with the normal control group, and significantly higher in the SASP $(\mathrm{P}<0.001)$ and decitabine groups $(\mathrm{P}<0.01)$ compared with the model group (Fig. 4B). However, differences in occludin expression among all groups were not statistically significant (Fig. 5A). The levels of ZO-1 and occludin in colon tissue of each group were detected by western blotting. The results demonstrated that the levels of $\mathrm{ZO}-1$ and occludin in the model group were significantly lower compared with those in the normal control group $(\mathrm{P}<0.001)$, indicating impaired colon barrier function. Compared with the model group, the expression levels of ZO-1 and occludin were significantly higher in the SASP and decitabine groups $(\mathrm{P}<0.001$; Fig. 5B).

Effect of decitabine on DSS-induced MAPK signaling pathway in colonic tissues of mice. The expression levels 
A

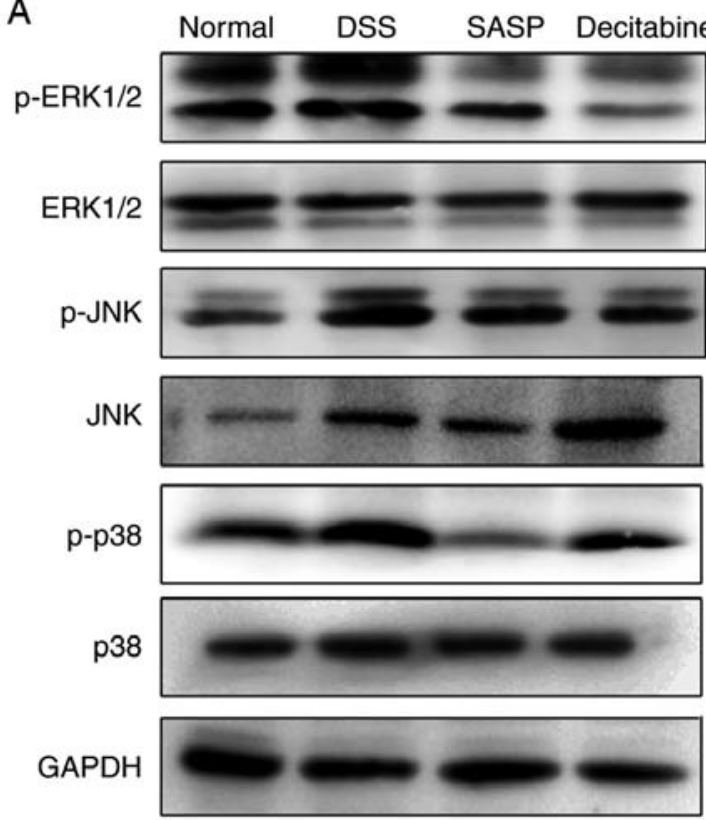

B



Figure 6. Effect of decitabine on DSS-induced MAPK signaling in colonic tissues of mice. (A) Western blot assay detected the levels of MAPK signaling-associated proteins in colonic tissues of mice. (B) The ratios of $\mathrm{p}$-protein/total protein in the model group were normalized to the norma group. All data are presented as mean \pm standard deviation and are representative of three independent experiments. ${ }^{*} \mathrm{P}<0.05,{ }^{* * *} \mathrm{P}<0.001$. DSS, dextran sulfate sodium; SASP, sulfasalazine; p-, phosphorylated.

of ERK1/2, JNK and p38 are presented in Fig. 6A. MAPK signaling is an important signaling system that utilizes a step-by-step phosphorylation process to amplify signals into the nucleus, regulate the activity of transcription factors and the expression of corresponding genes, and then cause cellular responses (24). It was determined that decitabine not only affected the expression of ERK1/2, JNK and P38 in the MAPK pathway, but also p-ERK1/2, p-JNK and p-p38. The phosphorylation of ERK1/2, JNK and p38 in each group is presented in Fig. 6B. Compared with the normal control group, the phosphorylation of ERK1/2, JNK and p38 protein in the model group were significantly increased $(\mathrm{P}<0.001)$, and significantly decreased after SASP $(\mathrm{P}<0.05)$ and decitabine $(\mathrm{P}<0.001)$ intervention. Phosphorylation of ERK1/2, JNK and $\mathrm{p} 38$ proteins represents the activation state of MAPK (25), and decitabine inhibits phosphorylation of ERK1/2, JNK and p38 in colon tissues, indicating that it can exert anti-inflammatory effects by inhibiting the activation of the MAPK signaling.

\section{Discussion}

$\mathrm{UC}$ is a chronic inflammatory disease of the rectum and colon that increases the risk of CRC in patients (26). UC is the predominant form of chronic IBD characterized by persistent inflammation in the mucosa and submucosa of the rectum and colon. UC is associated with the destruction of immune tolerance and leads to pathological inflammation (27).

The present study successfully established a DSS-induced colitis mouse model. The mice in the model group exhibited mucus, pus and bloody stools, weight loss. Gross morphology observation demonstrated obvious shortening of the colon, thickening of the intestinal wall and stenosis of the intestine. Congestion and edema were observed in the intestinal mucosa. A large amount of bloody secretions were observed in the intestine, accompanied by different degrees of erosion and ulcer formation. Under the microscope, the present study observed obvious congestion and edema, extensive large-scale erosion, deep ulcer formation, and disordered arrangement of crypts. A typical amount of colonic inflammation, such as lymphocyte and neutrophil infiltration, was observed in the mucosa and submucosa, indicating successful modeling. Subsequently, after 7 days of decitabine treatment, signs of diarrhea, mucus, pus and bloody stools in colitis mice were relieved, intestinal mucosal inflammation was reduced, and DAI, gross morphology and histological scores were significantly lower compared with the model group. These results indicate that decitabine has a good clinical effect on DSS-induced experimental colitis in mice.

Tregs are a subset of $\mathrm{CD}^{+} \mathrm{T}$ lymphocytes with inhibitory activity, which play an important role in controlling immune responses (28). A study has shown that IBD is associated with a decrease or dysfunction of Tregs (29). Effector T cells can induce intestinal hyperimmune response due to the lack of immunosuppressive regulation of Tregs, ultimately leading to intestinal mucosal damage (30). Tregs mainly secrete cytokines such as IL-4, IL-10 and TGF- $\beta 1$ (31). TGF- $\beta$, IL-2, IL-2R and IL-10 gene knockout mice can achieve spontaneous colitis due to the decrease of Tregs. Their intestinal tract exhibits crypt destruction, crypt abscess, submucosal inflammatory cell infiltration and other characteristics (32). Foxp3 is a major transcription factor that controls the development and function of Tregs. If Tregs (Foxp $3^{+} \mathrm{CD}^{+} \mathrm{T}$ cells) predominate over other pro-inflammatory $\mathrm{CD}^{+} \mathrm{T}$ cell subsets, the lesions are less severe (33). DNA methyltransferase inhibitor (DNMTi) 5-azacytidine and its derivative 5-aza 2'-deoxycytidine (decitabine) demonstrated immunomodulatory potential in vitro and in vivo, and induce demethylation of the Foxp3 gene (11). The application of DNMTi to induce Foxp3 expression has been extensively studied in mice (34). A previous study reported that decitabine is an inhibitor of DNMT, which blocks DNA methylation of the 5'-untranslated region promoter of Foxp3 and increases expression of Foxp3 in $\mathrm{CD}^{+}{ }^{+}$Foxp3 ${ }^{-}$cells $(35)$. Other preclinical studies have demonstrated that in vitro decitabine administration increases 
the population and immunosuppressive function of Foxp3 ${ }^{+}$ regulatory $\mathrm{T}$ cells (36). Landman et al (34) demonstrated that DNA methyltransferase can inhibit Th1 polarization in $\mathrm{CD}^{+}{ }^{+} \mathrm{CD} 25^{\text {high }} \mathrm{FOXP} 3^{+}$Tregs. Kehrmann et al (37) also reported that 5-aza-2'-deoxycytidine can induce Tregs. Epigenetic regulation of Foxp3 by DNMT can supply stable functional Tregs (28). Therefore, the present study suggested that decitabine can increase Tregs in vivo to treat UC. It was identified that the function of decitabine is dose-dependent. For decitabine, 'dual mechanism' refers to the inhibition of cell proliferation at high doses, and gene re-expression mediated by DNA hypomethylation at low doses, which affects cell differentiation and tumor suppression (38). The current study investigated whether $0.5 \mathrm{mg} / \mathrm{kg}$ decitabine could affect intestinal barrier function in mice with IBD by regulating naive $\mathrm{T}$ cell transformation in vivo. It was demonstrated that in $0.5 \mathrm{mg} / \mathrm{kg}$ decitabine-treated enteritis mice, the inflammatory response was inhibited, the release of the pro-inflammatory factor IL-17 was reduced and the release of the tumor suppressor IL-10 was promoted.

A decreased number of Tregs or dysfunction triggers IBD, meanwhile Th17 cells and their secreted cytokines also play an important role in the pathogenesis of IBD (39). Induction of Th17 differentiation leads to increased expression of IL-17 and results in decreased IL-10 expression (40). The highly expressed cytokine IL-17, which is activated by a Th17 and Tregs imbalance, synergizes with pro-inflammatory cytokines, such as TNF- $\alpha$ and IL-1 $\beta$, leading to a worsening of colitis (41). Therefore, inhibition of Th17 cell differentiation is considered a potential therapy for IBD (42). IL-17 is a major effector molecule of Th17 cells that exerts inflammatory effects. Neutralizing antibodies and small molecule inhibitors against IL-17 in different colitis animal models may exhibit different therapeutic effects (32). IL-10 is mainly secreted by Tregs and exerts an anti-inflammatory effect (43). IL-10 knockout and IL-10 receptor knockout mice spontaneously induce colitis (35). The present study identified that intraperitoneal injection of decitabine can promote the re-expression of Foxp3 in naive $\mathrm{T}$ cells in mice spleen, and increase the proportion of $\mathrm{CD}^{+}$Foxp $^{+} \mathrm{T}$ cells in $\mathrm{CD}^{+} \mathrm{T}$ cells, indicating that the use of DNMTi in vivo can play a role in demethylation and amplify Tregs. It also promotes IL-10 and TGF- $\beta$ and inhibits IL-17 expression.

The main connections between intestinal epithelial cells are tight junctions (44). Tight junctions are the determinant of intestinal barrier function; they can prevent antigens and microorganisms in the intestinal cavity from entering the body (45). The integrity of intestinal epithelial tight junctions is critical in IBD (44). The clinical symptoms of IBD are caused by intestinal inflammation and consequent dysfunction, including impaired absorption function and intestinal barrier function $(46,47)$. ZO-1 and occcludin are the main proteins constituting tight junctions, and changes in the levels of $\mathrm{ZO}-1$ and occcludin suggest a change of intestinal mucosal barrier function to a certain extent (48). Immunofluorescence in the present study revealed tight junction proteins in the decitabine group were distributed in the apical membrane of intestinal epithelial cells, with a continuous distribution and increased fluorescence intensity. Immunohistochemical results indicated that decitabine treatment can repair tight junction structures and microvilli, reduce intercellular gaps and cell vacuolation. Compared with the normal control group, the levels of ZO-1 and occludin in the intestinal epithelial cells of the model group were significantly decreased, indicating that the colon barrier function was impaired. Compared with the model group, the expression levels of ZO-1 and occludin in the SASP and decitabine groups were enhanced.

MAPK signaling is closely associated with the pathogenesis of UC, and its activation is considered to be one of the major factors leading to the release of cytokines and inflammatory mediators in UC (49). The MAPK pathway is an important signal transduction pathway involved in numerous biological functions. Abnormal activation of the MAPK pathway is closely associated with the overexpression of inflammatory cytokines and is also an important indicator for the occurrence and development of UC $(50,51)$. The MAPK family includes members such as ERK1, ERK2, JNK and p38 (52). Intervention of MAPK signaling can inhibit normal colonic epithelial cell apoptosis, accelerate colonic inflammatory cell apoptosis and inhibit the production of inflammatory cytokines, which is considered to be a potential anti-inflammatory molecular target for the treatment of UC (53). Phosphorylation of ERK1/2, JNK and p38 proteins represents the activation state of MAPK, and decitabine inhibited phosphorylation of ERK1/2, JNK and p38 in colon tissues, indicating that decitabine can exert anti-inflammatory effects by inhibiting the activation of the MAPK signaling.

In conclusion, the present study demonstrated that decitabine enhances the ratio of $\mathrm{CD}^{+}{ }^{+} \mathrm{Foxp}^{+} \mathrm{T}$ cells to $\mathrm{CD} 4^{+}$ $\mathrm{T}$ cells by promoting the re-expression of Foxp 3 in naive $\mathrm{T}$ cells in mice spleens, and amplifies Tregs, thereby repairing the colonic barrier of mice. Decitabine inhibits IL-17 expression in DSS-induced colitis mice and induces IL-10 and TGF- $\beta$, and thereby inhibits inflammation, which may be caused by inhibiting Th17 cell differentiation and promoting Treg differentiation. In addition, decitabine can also participate in anti-inflammatory effects by inhibiting the activation of the MAPK signaling.

\section{Acknowledgements}

Not applicable.

\section{Funding}

This study was supported by a grant from the Shanghai Minhang Hospital (grant no. 2018MHJC06).

\section{Availability of data and materials}

The datasets used and/or analysed during the current study are available from the corresponding author on reasonable request.

\section{Authors' contributions}

$\mathrm{JL}, \mathrm{PZ}$ and $\mathrm{YC}$ participated in the design of the study, interpretation of the results and review of the manuscript. CS, SL, XM and XY conducted the experiments. XM analyzed the data and wrote the manuscript. CS applied for the funds. All authors read and approved the final manuscript. 


\section{Ethics approval and consent to participate}

The animal research was approved by the Ethics Committee of Minhang Hospital, Shanghai, China [Medical Ethics Committee (2018) Approval no. 2].

\section{Patient consent for publication}

Not applicable.

\section{Competing interests}

The authors declare that they have no competing interests.

\section{References}

1. Yu SM and Kim SJ: 5-Azacytidine regulates matrix metalloproteinase-9 expression, and the migration and invasion of human fibrosarcoma HT1080 cells via PI3-kinase and ERK1/2 pathways. Int J Oncol 49: 1241-1247, 2016.

2. Woo JK, Choi S, Kang JH, Kim DE, Hurh BS, Jeon JE, Kim SY and Oh SH: BMC Complement Altern Med 16: 498, 2016.

3. Kim MW, Choi S, Kim SY, Yoon YS, Kang JH and Oh SH: Allyl isothiocyanate ameliorates dextran sodium sulfate-induced colitis in mouse by enhancing tight junction and mucin expression. Int J Mol Sci 19: pii: E2025, 2018.

4. Hartnett L and Egan LJ: Inflammation, DNA methylation and colitis-associated cancer. Carcinogenesis 33: 723-731, 2012.

5. Molodecky NA, Soon IS, Rabi DM, Ghali WA, Ferris M, Chernoff G, Benchimol EI, Panaccione R, Ghosh S, Barkema HW and Kaplan GG: Increasing incidence and prevalence of the inflammatory bowel diseases with time, based on systematic review. Gastroenterology 142: 46-54.e42, 2012.

6. Ramos GP and Papadakis KA: Mechanisms of disease: Inflammatory bowel diseases. Mayo Clin Proc 94: 155-165, 2019.

7. Scarpa M, Scarpa M, Castagliuolo I, Erroi F, Kotsafti A, Basato S, Brun P, D'Incà R, Rugge $\mathrm{M}$, Angriman I and Castoro C: Aberrant gene methylation in non-neoplastic mucosa as a predictive marker of ulcerative colitis-associated CRC. Oncotarget 7: 10322-10331, 2016.

8. Harris RA, Nagy-Szakal D, Mir SA, Frank E, Szigeti R, Kaplan JL, Bronsky J, Opekun A, Ferry GD, Winter H and Kellermayer R: DNA methylation-associated colonic mucosal immune and defense responses in treatment-naive pediatric ulcerative colitis. Epigenetics 9: 1131-1137, 2014.

9. Müller S, Fritz Y and Wagenknecht HA: Control of energy transfer between pyrene- and perylene-nucleosides by the sequence of DNA-templated supramolecular assemblies. ChemistryOpen 9: 389-392, 2020

10. Hollenbach PW, Nguyen AN, Brady H, Williams M, Ning Y, Richard N, Krushel L, Aukerman SL, Heise C and MacBeth KJ: A comparison of azacitidine and decitabine activities in acute myeloid leukemia cell lines. PLoS One 5: e9001, 2010.

11. Lu CH, Wu CJ, Chan CC, Nguyen DT, Lin KR, Lin SJ, Chen LC, Yen JJ and Kuo ML: DNA methyltransferase inhibitor promotes human CD4(+)CD25(h)FOXP3(+) regulatory T lymphocyte induction under suboptimal TCR stimulation. Front Immunol 7: 488, eCollection 2016, 2016.

12. Vargas-Robles H, Castro-Ochoa KF, Citalán-Madrid AF and Schnoor M: Beneficial effects of nutritional supplements on intestinal epithelial barrier functions in experimental colitis models in vivo. World J Gastroenterol 25: 4181-4198, 2019.

13. Sann H, Erichsen J, Hessmann M, Pahl A and Hoffmeyer A Efficacy of drugs used in the treatment of IBD and combinations there of in acute DSS-induced colitis in mice. Life Sci 92: 708-718, 2013.

14. Andújar I, Recio MC, Giner RM, Cienfuegos-Jovellanos E, Laghi S, Muguerza B and Ríos JL: Inhibition of ulcerative colitis in mice after oral administration of a polyphenol-enriched cocoa extract is mediated by the inhibition of STAT1 and STAT3 phosphorylation in colon cells. J Agric Food Chem 59: 6474-6483, 2011.

15. Nishiyama Y, Kataoka T, Yamato K, Taguchi T and Yamaoka K: Suppression of dextran sulfate sodium-induced colitis in mice by radon inhalation. Mediators Inflamm 2012: 239617, 2012.
16. Samak G, Chaudhry KK, Gangwar R, Narayanan D, Jaggar JH and Rao R: Calcium/Ask1/MKK7/JNK2/c-Src signalling cascade mediates disruption of intestinal epithelial tight junctions by dextran sulfate sodium. Biochem J 465: 503-515, 2015.

17. Yu J, Qin B, Moyer AM, Nowsheen S, Liu T, Qin S, Zhuang Y, Liu D, Lu SW, Kalari KR, et al: DNA methyltransferase expression in triple-negative breast cancer predicts sensitivity to decitabine. J Clin Invest 128: 2376-2388, 2018.

18. Kantarjian H, Issa PJ, Rosenfeld CS, Bennett JM, Albitar M, DiPersio J, Klimek V, Slack J, de Castro C, Ravandi F, et al: Decitabine improves patient outcomes in myelodysplastic Syndromes: Results of a phase III randomized study. Cancer 106: 1794-1803, 2006.

19. Cheung MC, Spalding PB, Gutierrez JC, Balkan W, Namias N, Koniaris LG and Zimmers TA: Body surface area prediction in normal, hypermuscular, and obese mice. J Surg Res 153: 326-331, 2009.

20. Jansen YJL, Verset G, Schats K, Van Dam PJ, Seremet T, Kockx M, Van Laethem JB and Neyns B: Phase I clinical trial of decitabine (5-aza2'-deoxycytidine) administered by hepatic arterial infusion in patients with unresectable liver-predominant metastases. ESMO Open 4: e000464, 2019.

21. Sakaguchi S, Miyara M, Costantino CM and Hafler DA: FOXP3+ regulatory $\mathrm{T}$ cells in the human immune system. Nat Rev Immunol 10: 490-500, 2010.

22. Danikowski KM, Jayaraman S and Prabhakar BS: Regulatory $\mathrm{T}$ cells in multiple sclerosis and myasthenia gravis. J Neuroinflammation 14: 117, 2017.

23. Nunes NS, Kim S, Sundby M, Chandran P, Burks SR, Paz AH and Frank JA: Temporal clinical, proteomic, histological and cellular immune responses of dextran sulfate sodium-induced acute colitis. World J Gastroenterol 24: 4341-4355, 2018.

24. Furumoto Y, Nunomura S, Terada T, Rivera J and Ra C: The FcepsilonRIbeta immunoreceptor tyrosine-based activation motif exerts inhibitory control on MAPK and IkappaB kinase phosphorylation and mast cell cytokine production. J Biol Chem 279: 49177-49187, 2004.

25. Marchesi N, Thongon N, Pascale A, Provenzani A, Koskela A, Korhonen E, Smedowski A, Govoni S, Kauppinen A, Kaarniranta K and Amadio M: Autophagy stimulus promotes early hur protein activation and p62/SQSTM1 protein synthesis in ARPE-19 cells by triggering Erk1/2, p38(MAPK), and JNK kinase pathways. Oxid Med Cell Longev 2018: 4956080-4956080, 2018.

26. Munkholm P: The incidence and prevalence of colorectal cancer in inflammatory bowel disease. Aliment Pharmacol Ther 18: 1-5, 2003.

27. Hou H, Cao R, Quan M, Sun Y, Sun H, Zhang J, Li B, Guo L and Song X: Rapamycin and fingolimod modulate Treg/Th17 cells in experimental autoimmune encephalomyelitis by regulating the Akt-mTOR and MAPK/ERK pathways. J Neuroimmunol 324: 26-34, 2018.

28. Wu CJ, Yang CY, Chen YH, Chen CM, Chen LC and Kuo ML: The DNA methylation inhibitor 5-azacytidine increases regulatory $\mathrm{T}$ cells and alleviates airway inflammation in ovalbumin-sensitized mice. Int Arch Allergy Immunol 160: 356-364, 2013.

29. Smids C, Horjus Talabur Horje CS, Drylewicz J, Drylewicz J, Roosenboom B, Groenen MJM, van Koolwijk E, van Lochem EG and Wahab PJ: Intestinal T cell profiling in inflammatory bowel disease: Linking $\mathrm{T}$ cell subsets to disease activity and disease course. J Crohns Colitis 12: 465-475, 2018.

30. Kaskow BJ and Baecher-Allan C: Effector T cells in multiple sclerosis. Cold Spring Harb Perspect Med 8: pii: a029025, 2018.

31. Ruitenberg EJ and Elgersma A: Response of intestinal globule leucocytes in the mouse during a Trichinella spiralis infection and its independence of intestinal mast cells. Br J Exp Pathol 60: 246-251, 1979.

32. Fitzpatrick LR: Inhibition of IL-17 as a pharmacological approach for IBD. Int Rev Immunol 32: 544-555, 2013.

33. Jacquemin C, Augusto JF, Scherlinger M, Gensous N, Forcade E, Douchet I, Levionnois E, Richez C, Lazaro E, Duffau P, et al: OX40L/OX40 axis impairs follicular and natural Treg function in human SLE. JCI Insight 3: pii: 122167, 2018.

34. Landman S, Cruijsen M, Urbano PCM, Huls G, van Erp PEJ, van Rijssen E, Joosten I and Koenen HJPM: DNA methyltransferase inhibition promotes th1 polarization in human CD4(+) CD25(high) FOXP3(+) regulatory T cells but does not affect their suppressive capacity. J Immunol Res 2018: 4973964, 2018. 
35. Fedorak RN, Gangl A, Elson CO, Rutgeerts P, Schreiber S, Wild G, Hanauer SB, Kilian A, Cohard M, LeBeaut A and Feagan B: Recombinant human interleukin 10 in the treatment of patients with mild to moderately active Crohn's disease. The Interleukin 10 Inflammatory Bowel Disease Cooperative Study Group. Gastroenterology 119: 1473-1482, 2000.

36. Polansky JK, Kretschmer K, Freyer J, Floess S, Garbe A, Baron U, Olek S, Hamann A, von Boehmer H and Huehn J: DNA methylation controls Foxp3 gene expression. Eur J Immunol 38: 1654-1663, 2008.

37. Kehrmann J, Tatura R, Zeschnigk M, Probst-Kepper M, Geffers R, Steinmann J and Buer J: Impact of 5-aza-2'-deoxycytidine and epigallocatechin-3-gallate for induction of human regulatory T cells. Immunology 142: 384-395, 2014.

38. Jabbour E, Issa JP, Garcia-Manero G and Kantarjian $\mathrm{H}$ : Evolution of decitabine development: Accomplishments, ongoing investigations, and future strategies. Cancer 112: 2341-2351, 2008.

39. Duan L, Rao X, Braunstein Z, Toomey AC and Zhong J: Role of incretin axis in inflammatory bowel disease. Front Immunol 8: $1734,2017$.

40. Hu Y, Cui Q, Gu Y, Sheng L, Wu K, Shi J, Tan Y, Fu H, Liu L, Fu S, et al: Decitabine facilitates the generation and immunosuppressive function of regulatory gammadeltaT cells derived from human peripheral blood mononuclear cells. Leukemia 27: $1580-1585,2013$.

41. Granet $\mathrm{C}$ and Miossec P: Combination of the pro-inflammatory cytokines IL-1, TNF-alpha and IL-17 leads to enhanced expression and additional recruitment of AP-1 family members, Egr-1 and NF-kappaB in osteoblast-like cells. Cytokine 26: 169-177, 2004.

42. Gizinski AM, Fox DA and Sarkar S: Pharmacotherapy: Concepts of pathogenesis and emerging treatments. Co-stimulation and $\mathrm{T}$ cells as therapeutic targets. Best Pract Res Clin Rheumatol 24: 463-477, 2010.

43. Brinkhoff A, Sieberichs A, Engler H, Dolff S, Benson S, Korth J, Schedlowski M, Kribben A, Witzke O and Wilde B: Pro-Inflammatory Th1 and Th17 cells are suppressed during human experimental endotoxemia whereas anti-inflammatory IL-10 producing T-cells are unaffected. Front Immunol 9: 1133, 2018.

44. Awad WA, Hess C and Hess M: Enteric pathogens and their toxin-induced disruption of the intestinal barrier through alteration of tight junctions in chickens. Toxins (Basel) 9: pii: E60, 2017.
45. Okumura $\mathrm{R}$ and Takeda K: Roles of intestinal epithelial cells in the maintenance of gut homeostasis. Exp Mol Med 49: e338, 2017.

46. Parikh K, Antanaviciute A, Fawkner-Corbett D, Jagielowicz M, Aulicino A, Lagerholm C, Davis S, Kinchen J, Chen HH, Alham NK, et al: Colonic epithelial cell diversity in health and inflammatory bowel disease. Nature 567: 49-55, 2019.

47. O'Callaghan AA and Corr SC: Establishing boundaries: The relationship that exists between intestinal epithelial cells and gut-dwelling bacteria. Microorganisms 7: pii: E663, 2019.

48. Vanhove W, Nys K, Arijs I, Cleynen I, Noben M, De Schepper S, Van Assche G, Ferrante M and Vermeire S: Biopsy-derived intestinal epithelial cell cultures for pathway-based stratification of patients with inflammatory bowel disease. J Crohns Colitis 12: 178-187, 2018.

49. Setia S, Nehru B and Sanyal SN: Upregulation of MAPK/Erk and PI3K/Akt pathways in ulcerative colitis-associated colon cancer. Biomed Pharmacother 68: 1023-1029, 2014.

50. Wagner EF and Nebreda AR: Signal integration by JNK and p38 MAPK pathways in cancer development. Nat Rev Cancer 9: 537-549, 2009.

51. You BH, Chae HS, Song J, Ko HW, Chin YW and Choi YH: $\alpha$-Mangostin ameliorates dextran sulfate sodium-induced colitis through inhibition of NF- $\mathrm{kB}$ and MAPK pathways. Int Immunopharmacol 49: 212-221, 2017.

52. Shematorova EK, Shpakovski DG, Chernysheva AD and Shpakovski GV: Molecular mechanisms of the juvenile form of batten disease: Important role of MAPK signaling pathways (ERK1/ERK2, JNK and p38) in pathogenesis of the malady. Biol Direct 13: 19, 2018.

53. Zhao HM, Huang XY, Zhou F, Tong WT, Wan PT, Huang MF, Ye Q and Liu DY: Si shen wan inhibits mRNA expression of apoptosis-related molecules in p38 MAPK signal pathway in mice with colitis. Evid Based Complement Alternat Med 2013: 432097, 2013.

This work is licensed under a Creative Commons Attribution-NonCommercial-NoDerivatives 4.0 International (CC BY-NC-ND 4.0) License. 